Gazi University
Journal of Science
http://dergipark.gov.tr/gujs

\title{
Effect of Downstream Expansion of a Long-Throated Flume on Flow Properties
}

\author{
Murat HATIPOGLU1 ${ }^{(1)}$, Mustafa GOGUS $^{2}$ (D), Muhammed $\mathrm{UCAR}^{3, *(D)}$ \\ ${ }^{I}$ General Directorate of State Hydraulics, Allocation Dept., 06000, Ankara, Turkey \\ ${ }^{2}$ Cankaya University, Civil Eng. Dept., 06000, Ankara, Turkey \\ ${ }^{3}$ Necmettin Erbakan University, Civil Eng. Dept., 42140, Konya, Turkey

\section{Highlights} \\ - Downstream transition type does not affect discharge coefficient - Froude number relation. \\ - There is a linear variation between discharge coefficient and Froude number. \\ - Downstream transition type does not affect the approach velocity coefficient.
}

\begin{tabular}{l} 
Article Info \\
\hline \\
Received: $27 / 02 / 2020$ \\
Accepted: $17 / 07 / 2020$ \\
Keywords \\
\hline Long-throated flumes \\
Downstream expansion \\
Modular limit \\
Discharge coefficient
\end{tabular}

\section{INTRODUCTION}

Measurement of flow in open channels is very important in the management of water. The flow rate can be measured by various structures. These structures, in general, are grouped as; weirs, orifices and flumes. The most widely used ones are the long throated flumes, Parshall flumes and H-flumes. Flow measurement structures designed especially for sediment-laden rivers are generally long-throated flumes or their altered types. [1]

Numerous studies conducted on various aspects of flow in expansion due to the significance of expansion applications. Nashta and Garde [2] investigated suddenly expanded subcritical upstream flow in rectangular rigid bed channels with expansion ratios of 1.5 to 3.0 and presented analytical and experimental results. They showed that the energy loss coefficient increases with increasing expansion ratios. Swamee and Basak [3] offered a method for the design of expansions with trapezoidal transition channel that connect a rectangular channel section for subcritical flow. They suggested an empirical formulation for channel transition shape and claimed that the associated energy losses were considerably reduced with minimum flow separation.

A number of studies exist both experimental and computational on supercritical flows in gradual expansions concerning surface profiles, velocities, shock wave formation and properties. The results obtained from the numerical methods were compared to experimental data and a satisfactory agreement was obtained in most 
cases (Bhallamudi \& Chaudhry [4]; Krueger \& Rutschmann [5]; Mazumder \& Hager [6]; Stamou et al. [7]). Gogus et al. [8] conducted laboratory tests on nine flow measurement models of rectangular compound cross section having different downstream transitions. The effect of the downstream expansion region of the models on discharge coefficient $C_{d}$, the approach velocity coefficient $C_{v}$, and the modular limit $\left(H_{2} / H_{1}\right)_{M L}$ were investigated. It was concluded that the model having a downstream slope of about 1/7 yielded the highest modular limit. Nasser and Li [9] carried out analytical and experimental studies for subcritical flows passing through expansions and the authors shown that the simple triangular hump fitted at the bottom of the flat-bottom expansion is effective in reducing energy head losses. Asnaashari et al. [10] presented a flow field investigation in a gradual transition of rectangular to trapezoidal open channels, test were conducted both numerically and experimentally. The authors also investigated the effect of the different inlet discharges on the separation zones and at the transition corners and the secondary currents were also investigated.

The aim of this experimental study was, in short, to analyse the effect of the type of downstream transition in a long-throated flume on the hydraulic properties of the flow. In this study, a long throated flume of rectangular cross section with various downstream expansions was experimentally analysed at the laboratory. Extensive experiments were conducted on the structure for five different downstream expansions. The effect of the downstream expansion on the submergence ratios of flow, modular limit, on the flow pattern in the energy dissipater and some other hydraulic quantities were investigated.

\section{MATERIAL METHOD}

The study is conducted on experimental setup with five different downstream expansions by the guide of dimensional analysis results. The dimensional analysis is given in the theoretical background subsection. Of all known flumes and weirs "long-throated flume" has certain advantages some of which are as follows (Figure 1) [1];

a) In all these types of flumes at the downstream section of the structure that is called as "control section", critical flow is formed by a contraction of the river width and/or a drop of the river bottom. The control section is followed by a transition to the energy dissipater where the flow becomes subcritical.

b) The throat section can have a wide variety of shapes

c) From existing hydraulic theory, the head loss over the flume for a unique upstream headdischarge relation can be approximated with sufficient accuracy.

d) The structure can be designed to pass sediment transported by channels. 


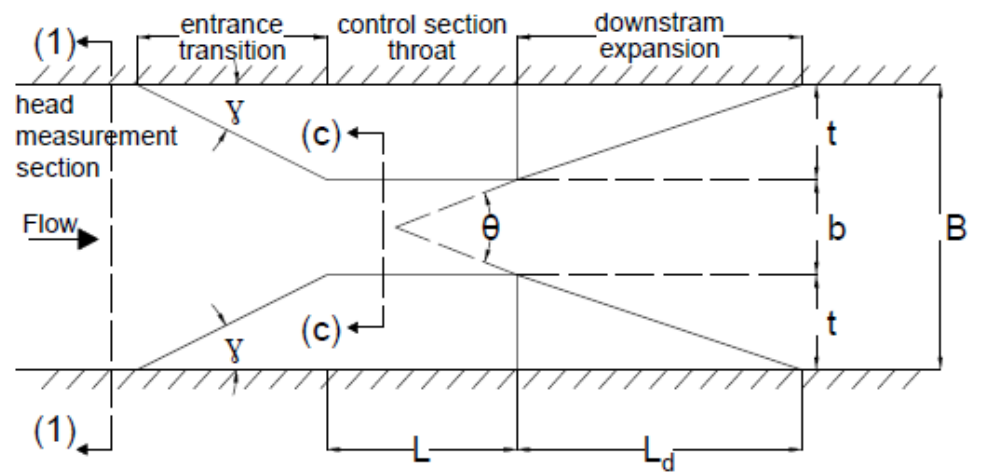

a) Plan View

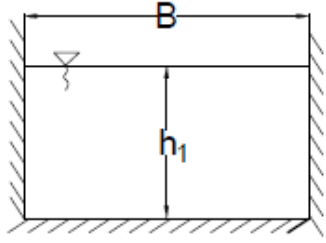

c) section (1)-(1)

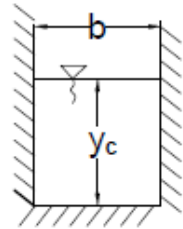

d) section (c)-(c)

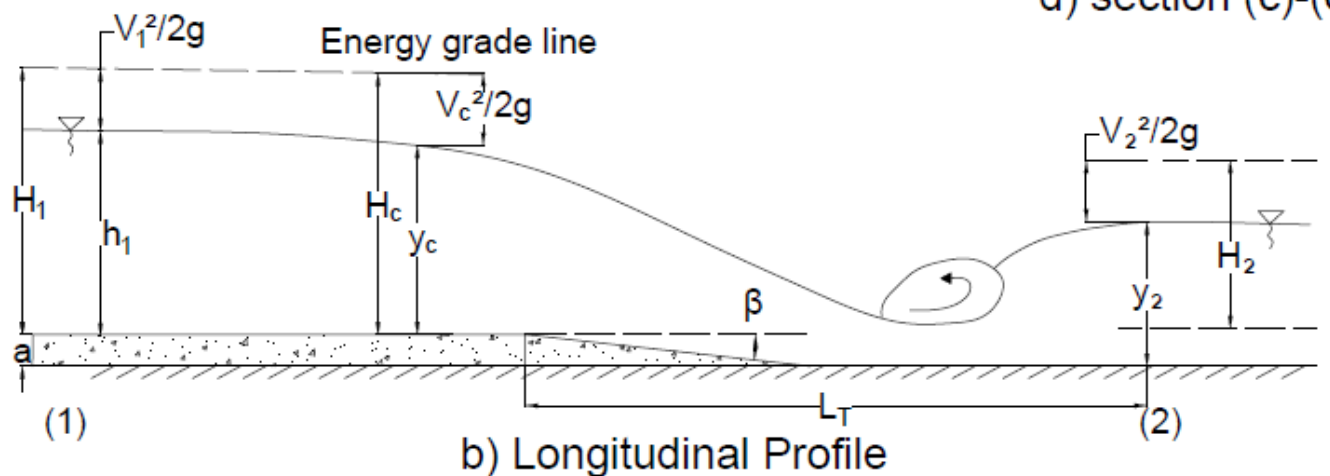

Figure 1. Definition sketch of the long-throated flume of rectangular cross-section used in this study

In a long-throated flume, the flow becomes critical in the throat and then continues as supercritical flow through the downstream expansion region after which the flow turns into subcritical flow. The magnitude of the energy loss, which occurs over the downstream expansion length, is very important when the submergence ratio of the flume is concerned. As stated by Bos [1], in 1955 G.Formica conducted experiments in various open channel expansions for subcritical flow conditions and obtained energy loss coefficients, $K_{L}$ from the equation given below

$$
\Delta H=K_{L} \frac{\left(V_{1}-V_{2}\right)^{2}}{2 g}
$$

where, $\Delta H$ is the energy loss per unit weight of the flowing fluid between sections (1) and (2) depicted in Figure $2, K_{L}$ is the energy loss coefficient, $V_{1}$ and $V_{2}$ are the average flow velocities at sections given by subscripts, and $g$ is the acceleration of gravity [1].

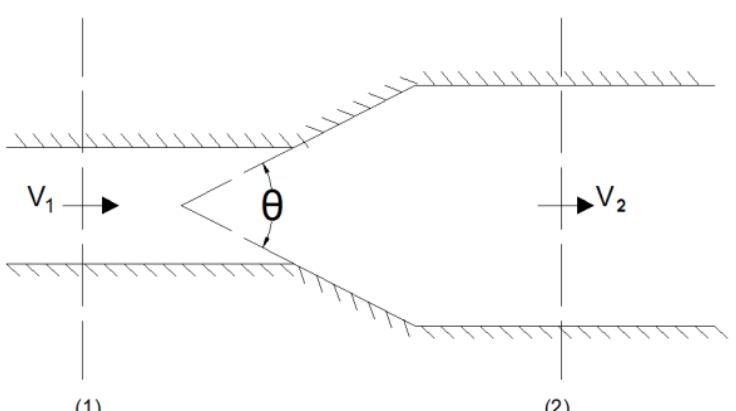

Figure 2. Definition sketch for channel expansion 


\subsection{Theoretical Background}

Figure 1 shows the plan view and the longitudinal profile of a long-throated flume of rectangular crosssection. Between section (1) and control section (c) where the flow is critical, assuming that all types of energy losses are negligible, velocity distributions are uniform and streamlines all straight and parallel to each other, one can write the energy equation as;

$$
H_{1}=h_{1}+\frac{V_{1}^{2}}{2 g}=y_{C}+\frac{V_{c}^{2}}{2 g}
$$

where $H_{1}$ is the total (specific) energy head per unit weight of the flowing fluid, $h_{1}$ is the flow depth, $V_{1}$ is the average flow velocity, $g$ is the acceleration of gravity, $y_{c}$ is the critical flow depth and $V_{c}$ is the critical flow velocity.

Applying the continuity equation to head measurement and control sections and combining it with Equation (2) and the relation of critical flow depth for rectangular cross-sections, the relation for discharge $Q$, Equation (3), can be written as

$$
Q=\frac{2}{3} C_{d} b\left(\frac{2}{3} g\right)^{1 / 2} H_{1}^{3 / 2}
$$

where $C_{d}$ is the discharge coefficient introduced to account for the idealized assumptions made in the derivation of Equation (2).

It is not possible to measure the energy head $H_{1}$ in directly in a field installation and therefore commonly upstream water level over the crest $h_{1}$ is related to $H_{1}$ by seemingly neglecting the velocity head $V_{1}^{2} / 2 g$ at the measurement section, whilst it is corrected by the approach velocity coefficient $C_{v}$. Then, Equation (3) becomes

$$
Q=\frac{2}{3} b C_{d} C_{v}\left(\frac{2}{3} g\right)^{1 / 2} h_{1}^{3 / 2}
$$

In this equation, $C_{d}$ is the discharge coefficient which is generally related to the dimensionless ratio $H_{1} / L$ where $L$ is the throat length.

The effect of the downstream expansion of long throated flumes on the discharge coefficient $C_{d}$ needed to be investigated due to the influence of the type on streamline curvature of the flow at the control section. For this reason, the variation of $C_{d}$ with $H_{1} / L$ values for each downstream expansion type tested will be presented in the related section.

As for the approach velocity coefficient $C_{v}$, from the studies have been conducted so far, it is known that $C_{v}$ is expressed as a function of the term $\sqrt{\alpha} C_{d} A^{*} / A_{1}$ with the type of the control section as a third variable [11]. Here $\alpha$ is the approach velocity distribution coefficient (usually approximated by 1.04 ), $A^{*}$ is the imaginary wetted area at the control section if the water depth would equal to $\mathrm{h}$ (for rectangular control sections $\left.A^{*}=b h_{1}\right), A_{1}$ is the wetted area at the flow depth measurement section and $\mathrm{u}$ is the power of $h_{1}$ in the head discharge equation and is related to the shape of the control section.

Since $C_{v}$ is related to $C_{d}$ in addition to other terms and as it had been stated that $C_{d}$ would be influenced by the downstream expansion of the structure, the relation between $C_{v}$ and the type of the downstream expansions will be investigated in this study, too.

The difference between the total energy head of the flow at depth measurement section $H_{1}$, and the total energy head of the flow at the energy dissipater after the jump $\mathrm{H}_{2}$, is the available energy loss over the structure. The ratio of $H_{2}$ to $H_{1}$ is known as "submergence ratio". For low values of $H_{2} / H_{1}$, the tail water parameters will not affect the upstream flow conditions. In this case, a unique relation can be obtained between the flow depth $h_{1}$ and $Q$, and the flow through the channel is called "modular" or "free". When the 
ratio of $H_{2} / H_{1}$ exceeds a certain value, the flow over the structure becomes "submerged" or "non-modular" and the unique relation between $h_{1}$ and $Q$ cannot be achieved. This critical value of $H_{2} / H_{1}$ is called as "critical submergence ratio" or "modular limit", at which the real discharge rate deviates by $1 \%$.

The modular limit required to be known if the channel is to be designed in the modular flow range. This limit was determined for the tested flumes of this study by applying the head discharge equation presented in Equation (3) to the definition of modular limit given above

$$
\frac{Q_{(101)}}{Q_{(100)}}=\frac{\frac{2}{3} C_{d} b\left(\frac{2}{3} g\right)^{1 / 2} H_{1(101)}^{3 / 2}}{\frac{2}{3} C_{d} b\left(\frac{2}{3} g\right)^{1 / 2} H_{1(100)}^{3 / 2}}
$$

from which

$$
1.01=\left(\frac{H_{1(101)}}{H_{1(100)}}\right)^{3 / 2}
$$

or

$$
H_{1(101)}=1.0067 H_{1(100)} \text {. }
$$

After determining $H_{1(101)}$ from Equation (7), the value of $h_{1(101)}$, which is the flow depth to be formed at the depth measurement section for which the flow over the long-throated flume will have critical submergence ratio, can be computed from Equation (8)

$$
h_{1(101)}=H_{1(101)}-\frac{V_{1}^{2}}{2 g} \text {. }
$$

During experiments to be conducted at the laboratory for flow conditions of known $H_{1(100)}$; from Equations (7) and (8) first $H_{1(101)}$ and then $h_{1(101)}$ are to be calculated, and the tail water gate of the flume will be very slowly lifted until the flow depth at the depth measurement section becomes equal to $h_{1(101)}$. When this situation is achieved, the tail water gate is to be fixed and the required flow measurements will be done.

In this study the most important dependent parameters for which the relations between the relevant dimensionless terms to be searched for are; the discharge coefficient $C_{d}$, the modular limit of the flow $H_{2} / H_{1}$ and the length of the energy dissipater which also covers the downstream transition length $L_{T}$. Figure 1 shows the definition sketch of the structure and quantities used in the experiments and dimensional analysis, respectively.

The discharge coefficient $C_{d}$, as had been stated before, compensates for the errors to be made from the assumptions utilized in the derivation of discharge-head relation. One can express the value of $C_{d}$ in the following form as a function of the independent variables involved in the phenomena

$$
C_{d}=f\left(h_{1}, V_{1}, a, g, B, b, L, \beta, \gamma, \theta, k\right)
$$

where $a$ is the step height, $B$ is the main channel with, $b$ is the throat with, $L$ is the throat length, $\beta$ is the vertical angle between throat bed form and the downstream transition, $\gamma$ is the angle between the channel wall and the upstream transition, $\theta$ is the downstream expansion of the system and $k$ is the characteristic roughness height of the channel.

Since the independent quantities stated in Equation (9) are the predominant ones to be referred in dimensional analysis, other dependent variables indicated at the beginning of this section, that is, modular limit $\left(H_{2} / H_{1}\right)_{M L}$, the total length of the energy dissipater $L_{T}$, which are temporarily designated as $(D . V)_{1}$, can also be written in the form of Equation (9) as 


$$
\left\{\begin{array}{c}
\left(H_{2} / H_{1}\right)_{M L} \\
L_{T} \\
C_{d}
\end{array}=(D . V)_{1}=f\left(h_{1}, V_{1}, a, g, B, b, L, \beta, \gamma, \theta, k\right) .\right.
$$

Selecting $h_{1}$ and $g$ as repeating variables, the following dimensionless quantities are obtained from Equation (10)

$$
(D . V)_{2}=f\left(\frac{V_{1}}{\sqrt{g h_{1}}}, \frac{a}{h_{1}}, \beta, \gamma, \theta, \frac{b}{B}, \frac{L}{h_{1}}, \frac{k}{h_{1}}\right) .
$$

where (D.V) $)_{2}$ stands for dependent variables $C_{d},\left(H_{2} / H_{1}\right)_{M L}$ and $L_{T} / h_{1}$. Throughout this study $a, L, B, b$ and $\gamma$ were kept constant due to the time and source limitation of the study. $\beta$ and $\theta$ values are specified for five model types (Table 1), one using the given graphs at the discussion section should once select the model type relevant to one's own particular case, interpolation may also be needed for intermediate values. For a preselected type of the model, $\beta$ and $\theta$ values are fixed as well as $b / B, \gamma$ and $k / h_{1}$, which is the relative roughness height of the channel. Since in the derivation of the theoretical equation of discharge all of the frictional losses had been assumed to be zero, $k / h_{1}$ and the other constant dimensionless terms stated above can be removed from Equation (11). $\frac{V_{1}}{\sqrt{g h_{1}}}$ stands for upstream Froude number, $F r_{1}$. In this case,

$$
(D . V)_{2}=f\left(F r_{1}, \frac{a}{h_{1}}, \frac{L}{h_{1}}\right)
$$

or in the form of Equation (13), by replacing $h_{1}$ with $H_{1}$ in the parameter of $L / h_{1}$, and introducing this related dimensionless term as $H_{1} / L$, to consider the effect of approach velocity head into the analysis

$$
(D . V)_{2}=f\left(F r_{1}, \frac{a}{h_{1}}, \frac{H_{1}}{L}\right) .
$$

\subsection{Experimental Investigation}

All series of experiments were performed in a glass walled horizontal laboratory flume of $12 \mathrm{~m}$ in length, $0.45 \mathrm{~m}$ in width and $0.75 \mathrm{~m}$ in depth in the Hydromechanics Laboratory of the Middle East Technical University.

The long-throated flume of fixed dimensions without a downstream transition was manufactured from thin steel plates and placed to the mid-length of the main channel. Five types of downstream transition was prepared from concrete and steel plates and mounted to the channel. The entire model types tested consists of the following parts: approach channel, converging transition, throat, diverging transition and tail water channel (Figure 1). The dimensions of the long-throated flumes used in the experiments are given in Table 1 .

Table 1. Dimensions of the types of long-throated flumes tested (Figure 1)

\begin{tabular}{lllllllllllllll}
\hline $\begin{array}{l}\text { Model } \\
\text { type }\end{array}$ & $\begin{array}{l}\theta \\
(\mathrm{deg})\end{array}$ & $\beta(\mathrm{deg})$ & $\gamma(\mathrm{deg})$ & $a$ & $(\mathrm{~cm})$ & $B$ & $(\mathrm{~cm})$ & $b$ & $(\mathrm{~cm})$ & $L$ & $(\mathrm{~cm})$ & $t$ & $(\mathrm{~cm})$ & $\begin{array}{l}L_{d} \\
(\mathrm{~cm})\end{array}$ \\
\hline 1 & 180.0 & 90 & 26.57 & 5 & 45 & 15 & 30 & 15 & 0 \\
2 & 112.62 & 26.57 & 26.57 & 5 & 45 & 15 & 30 & 15 & 10 \\
3 & 73.74 & 14.04 & 26.57 & 5 & 45 & 15 & 30 & 15 & 20 \\
4 & 53.13 & 9.46 & 26.57 & 5 & 45 & 15 & 30 & 15 & 30 \\
5 & 41.11 & 7.13 & 26.57 & 5 & 45 & 15 & 30 & 15 & 40 \\
\hline
\end{tabular}

The discharge was determined with a rectangular sharp crested weir mounted upstream part of the main channel. Measurement of head over the crest for this weir was done by using a point gauge. 
In the head measurements, 2 point gauges with an accuracy of $\pm 0.1 \mathrm{~mm}$ were used. The first one was placed to the head measurement section of the approach channel to measure the upstream head $h_{1}$. The other one was movable for measuring critical depth $y_{c}$ and the flow depth $y_{2}$ at the throat and tail water channel, respectively. All depths mentioned above were measured with respect to the crest elevation.

Experiments were performed first for free flow cases and then submerged flow (modular limit) cases.

In the experiments, five types of models with various downstream sections were tested in a row. For a selected type of the model; first, the tail water gate of the main channel was kept fully open and then the minimum measurable flow discharge was conveyed through the model. The flow discharge $Q$, the upstream flow depth $h_{1}$ were measured. After that for the same flow discharge, the tail water depth was raised by lifting the tail water gate of the main channel to create a hydraulic jump at the downstream section of the model. For this situation the flow depth $y_{2}$ in the tail water channel after the hydraulic jump and the total required length of the energy dissipater $L_{T}$, were measured.

The measurements stated above were repeated for a very wide range of flow discharges which would be possible to supply from the constant-head water storage of the laboratory. The ranges of some hydraulic characteristics are; discharge $(Q)$ between $4.76 \mathrm{lt} / \mathrm{s}-47.15 \mathrm{lt} / \mathrm{s}$, upstream flow depth $\left(h_{1}\right)$ between $7.55 \mathrm{~cm}$ - $31.90 \mathrm{~cm}$, upstream Froude number $\left(F r_{1}\right)$ between 0.159 - 0.187 .

After completing the free flow measurements, the point gauge fixed at the head measurement section was raised up to the value of $h_{1(101)}$. By lifting the tail water gate gradually, the tail water depth of the flow was increased so that at the depth measurement section the water surface touched the point gauge. At this stage, downstream head $y_{2}$ was measured by a movable point gauge. Then corresponding $\mathrm{H}_{2}$ and modular limit $H_{2} / H_{1(101)}(101)$ were calculated. The minimum required length of the energy dissipater corresponding to the modular limit case was also recorded.

\section{DISCUSSION OF THE RESULTS}

\subsection{The Discharge Coefficient $C_{d}$}

In order to see the effect of $H_{1} / L$ on the values of discharge coefficient $C_{d}$ for each of the model types tested Figure 3 was plotted. The analysis of the data shows that the effect of the model type on the $C_{d}$ values cannot be clearly stated. The distribution of the data points is quite random, however, it can be concluded that $C_{d}$ increases as $H_{1} / L$ increases. The reason of this situation is that as $H_{1} / L$ value increases, the effect of flow curvature over the control section on the discharge becomes less important.

To make a comparison between the distribution of $C_{d}$ data presented by Bos [1] and those given in Figure 3 , only the best fitting curve of the data points obtained by various investigators and given by Bos [1] was plotted on Figure 3. At this point, it should be stated that almost 50\% of the points lie above the Bos's best fitting curve while the remaining 50\% lies below it. Figure 3 shows that even though most of the present data fall below the best fitting curve of Bos [1], almost $50 \%$ of the data coincides with the data of other investigators. 


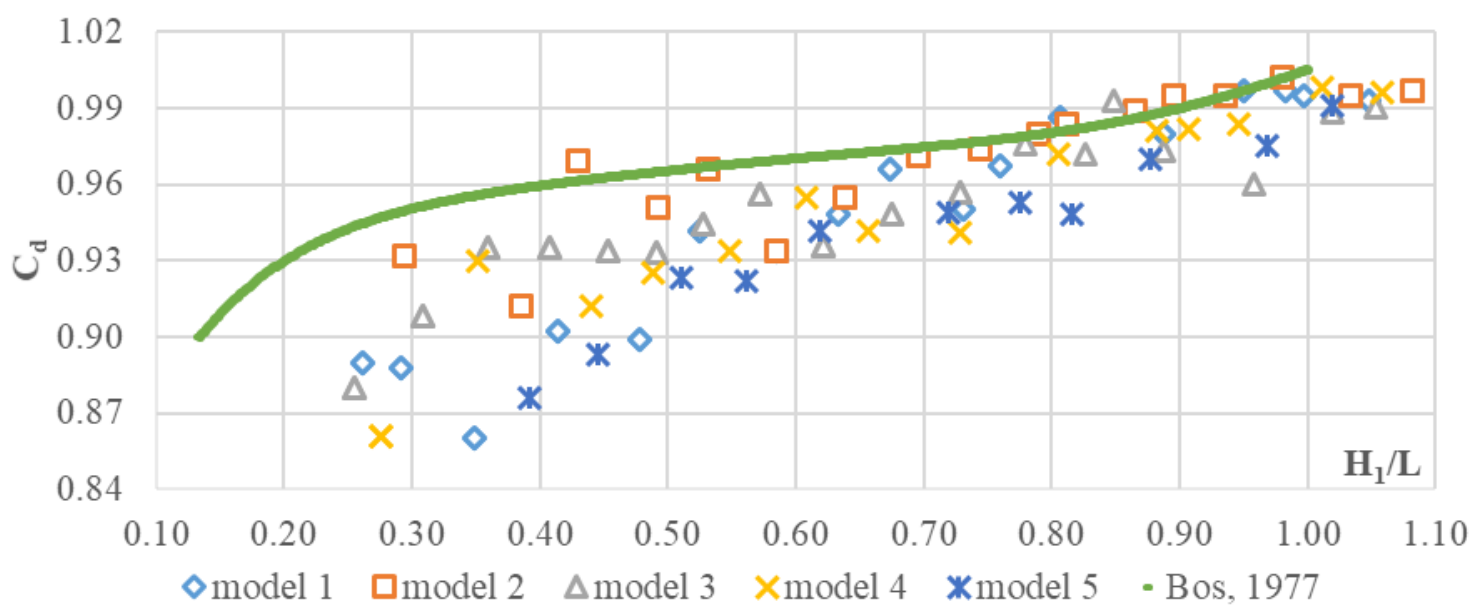

Figure 3. Variation of discharge coefficient with $\boldsymbol{H}_{\mathbf{1}} / \mathbf{L}$

Figure 4 presents the variation of $C_{d}$ with $F r_{1}$ for model types of 1-5. Data points in each model fall on a straight line. No attempt was made to give the equation of the best fitting line considering that the number of data was not enough to generalize the experimental results which are only valid for the flow conditions tested in this study. Consequently, one can conclude that there is a single linear relationship between $C_{d}$ and $F r_{1}$ whatever the type of the model is. When the upstream flow conditions are known such as $h_{1}$ and $\mathrm{V}_{1}$, one can easily determine the corresponding $C_{d}$ value from Figure 4.

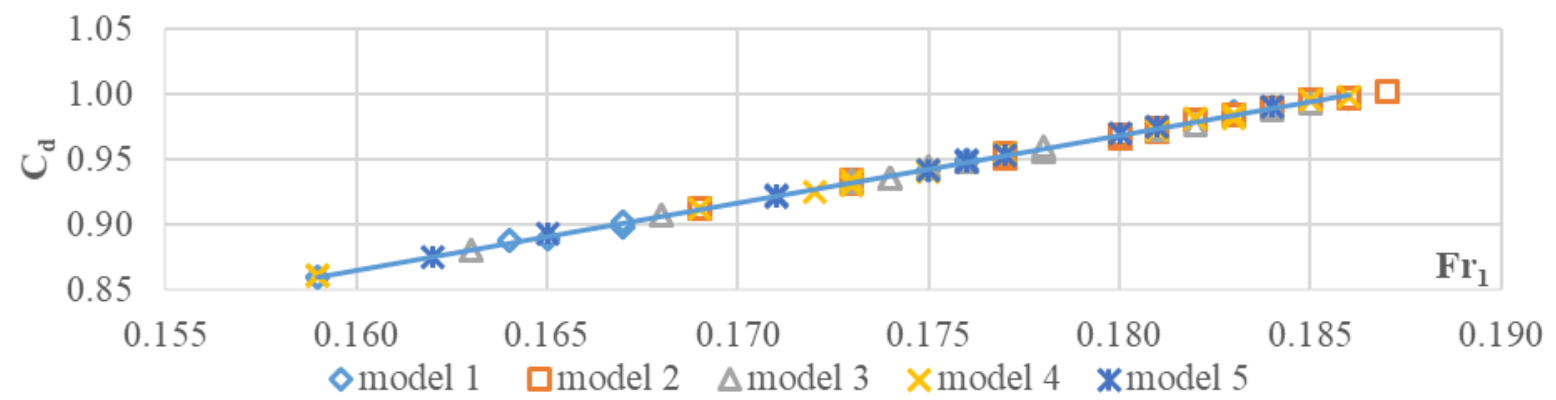

Figure 4. Variation of discharge coefficient with Froude number for model types tested

\subsection{The Approach Velocity Coefficient $C_{v}$}

As stated earlier the approach velocity coefficient, in general, was presented as a function $\sqrt{\alpha} C_{d} A^{*} / A_{1}$, and the related data of the models tested are shown in Figure 5. From the general trends of the data given in Figure 5 it can be stated that there is almost no influence of the model type on $C_{v}$ values for a given value of $\sqrt{\alpha} C_{d} A^{*} / A_{1}$.

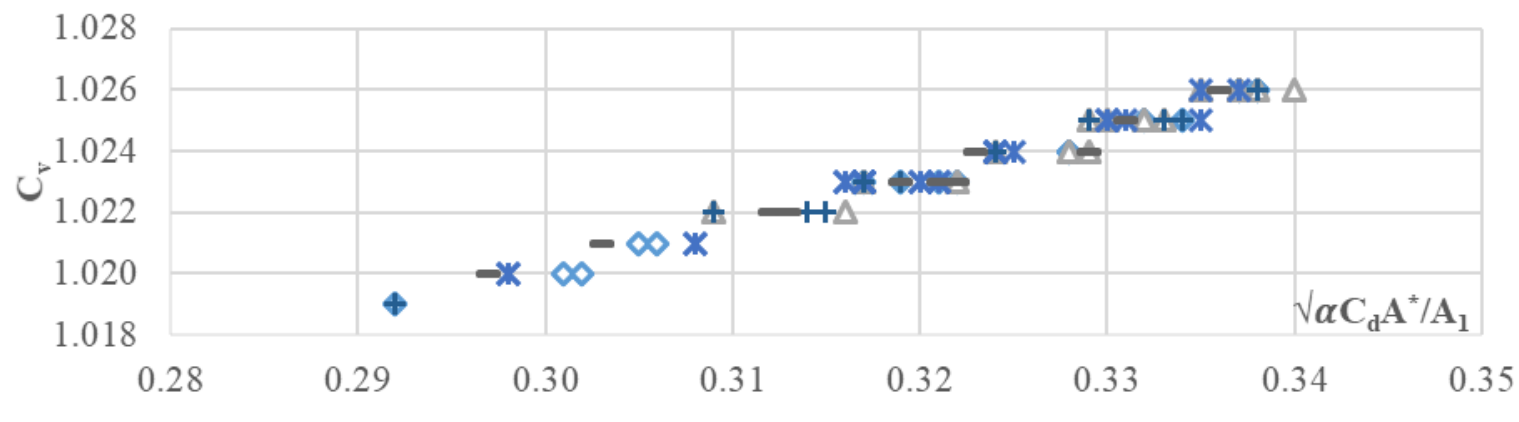

$\diamond$ model $1 \Delta$ model $2 \quad$ *model $3 \quad+$ model 4 -model 5

Figure 5. Variation of approach velocity coefficient with $\sqrt{\alpha} C_{d} A^{*} / A_{1}$, for all the model types tested 


\subsection{Variation of $K_{L}$ with Angle of Expansion $\theta$ and $H_{1} / L$}

The variation of the energy loss coefficient $K_{L}$ with the downstream expansion angle of $\theta$ was presented in Figure 6. In this figure, all of the $K_{L}$ values obtained from free flow and modular limit situations were plotted. This figure reveals that $K_{L}$ values of free flows for a given model are always much smaller than those of modular flows. Model type 1 which has an abrupt expansion, $\theta=180^{\circ}$, yields the maximum energy losses as expected. As the value of $\theta$ decreases, that is the length of the expansion zone of the channel $L_{d}$ increases, $K_{L}$ values decrease.

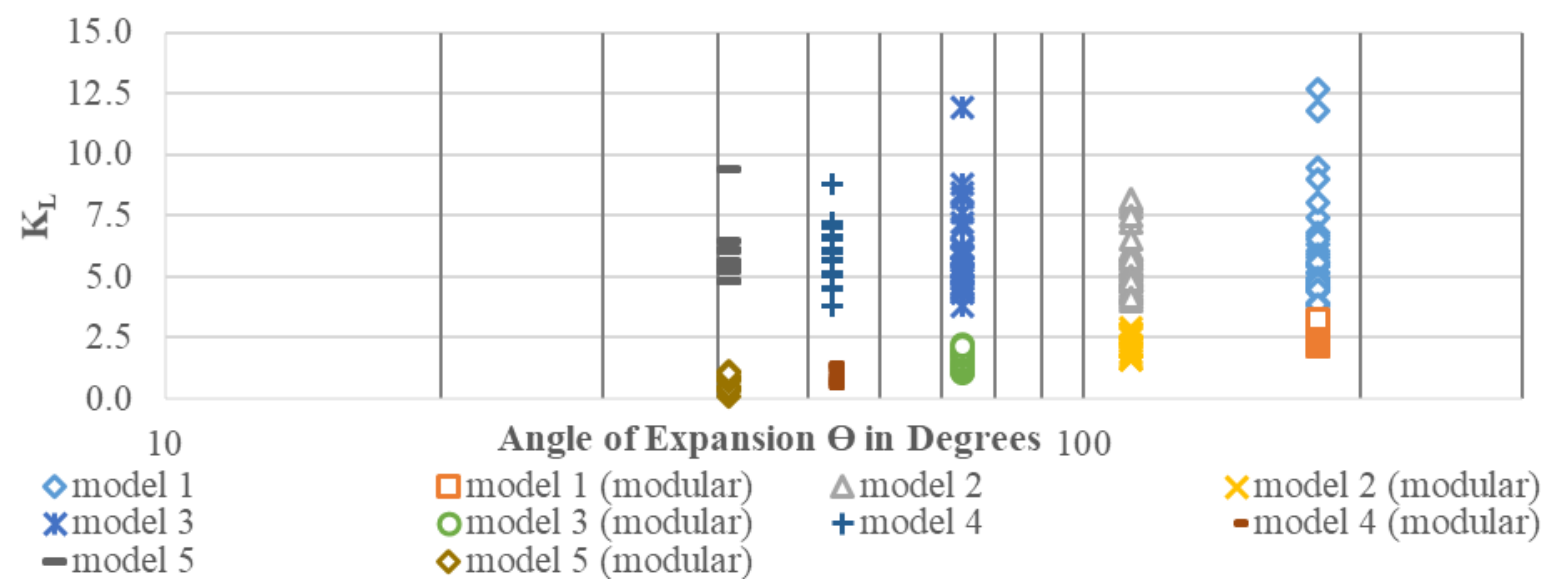

Figure 6. Variation of energy loss coefficient with downstream expansion angle of $\theta$ for free flow and modular limit cases of model types tested

If one plots only the data of $K_{L}$ for modular limit cases as a function of $\theta$, Figure 7 is obtained. $K_{L}$ values of model types of 4 and 5 are less than 1.5 while the other model types have $K_{L}$ values between 1.0 and 3.5. This situation implies that as the values $\theta$ get smaller, $K_{L}$ values decrease and therefore higher modular limits are provided.

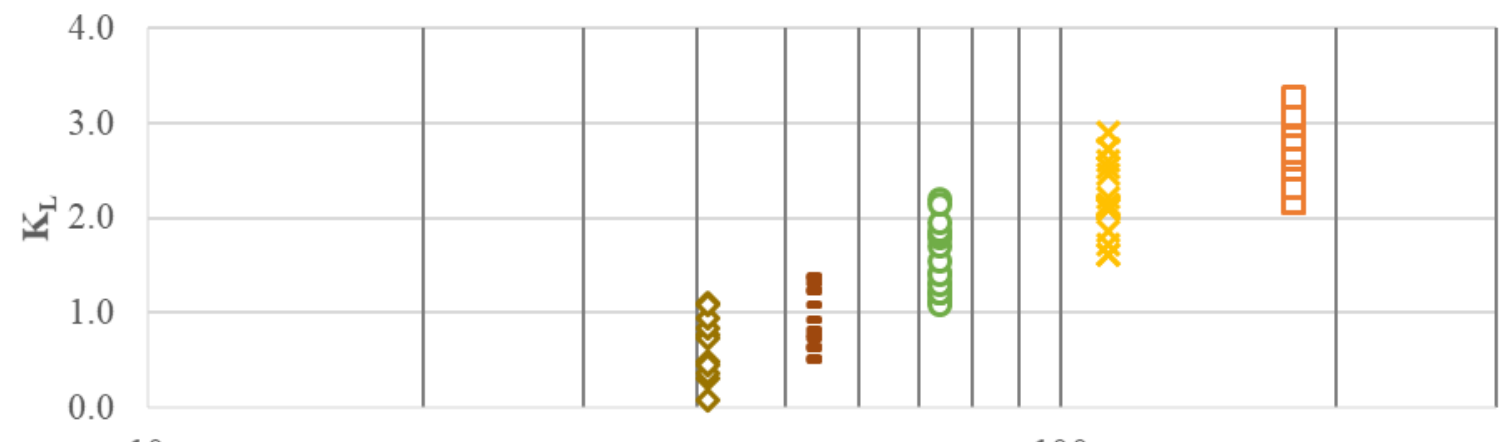

10

Angle of Expansion $\boldsymbol{\theta}$ in Degrees 100

$\square$ model 1 (modular) $\times$ model 2 (modular) Omodel 3 (modular) - model 4 (modular) $\diamond$ model 5 (modular)

Figure 7. Variation of energy loss coefficient with downstream expansion angle of $\Theta$ for modular limit cases

Figure 8 shows the variation of $K_{L}$ with $H_{1} / L$ for the model types tested. From the general trends of the data points for each model type it is seen that $K_{L}$ values increase as $H_{1} / L$ gets larger. For the same $H_{1} / L$, model types 1 and 5 give the highest and lowest $K_{L}$ values, respectively. It means that critical depth flumes to be constructed with $\theta$ values smaller than that of model 5 will always result in small $K_{L}$ values but higher modular limits. 


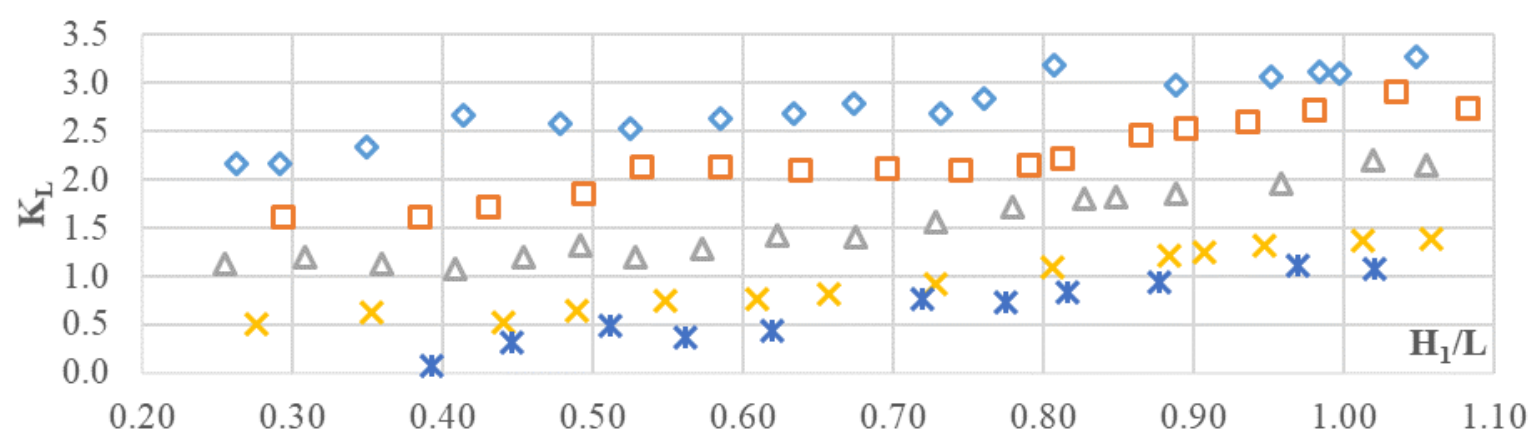

$\diamond$ model 1 (modular) $\square$ model 2 (modular) $\Delta$ model 3 (modular) $\times$ model 4 (modular) $*$ model 5 (modular)

Figure 8. Variation of energy loss coefficient with dimensionless ratio $\boldsymbol{H}_{\mathbf{1}} / \mathbf{L}$ for modular limit cases

\subsection{The Modular Limit}

To determine the modular limit of a given model type for a known value of $F r_{1}$, the calculated values of modular limits of each model type were plotted as a function of $\mathrm{Fr}_{1}$ and presented in Figure 9. In each model type, modular limit values slightly decrease as $F r_{1}$ increases, except model type 1 for which $\left(H_{2} / H_{1}\right)_{M L}$ is almost constant for the range of $F r_{1}$ tested. This is due to the increasing $K_{L}$ values as $H_{1} / L$ values (or $F r_{1}$ ) increase (Figure 8). The model types 1 and 5 give the lowest and highest $\left(H_{2} / H_{1}\right)_{M L}$ values, respectively, for a given $F r_{1}$. Among the model types tested the type 5 attains a modular limit value as high as 0.90 . It is obvious that the long throated critical depth flumes to be built with an angle of $\theta$ smaller than that of model type 5, will yield a modular limit in between 0.90 and 1.0. By means of Figure 9 one can easily determine $\left(H_{2} / H_{1}\right)_{M L}$ for a given model type and $F r_{1}$.

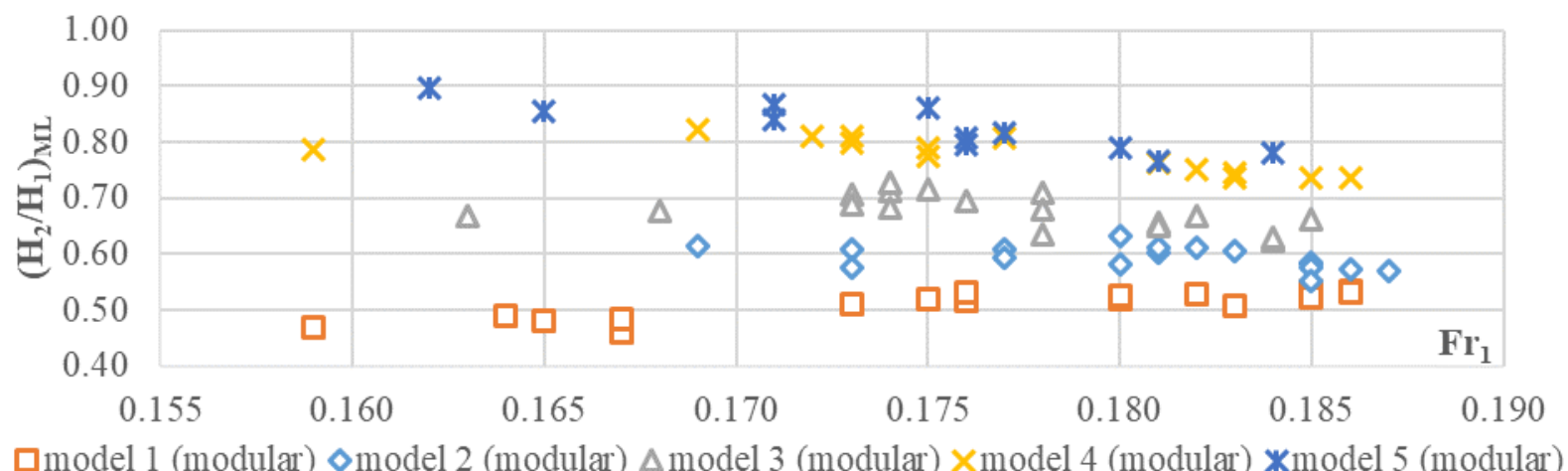

Figure 9. Variation of Modular limit with Froude number

The variation of modular limit with $a / h_{1}$ for each model type tested was presented in Figure 10.Modular limit values slightly increase or decrease as $a / h_{1}$ increases in the model types tested. One may also roughly say that the effect of $a / h_{1}$ on the distribution of $\left(H_{2} / H_{1}\right)_{M L}$ is almost negligible, except the model type 5, which tends to increase more rapidly than the other model types as $a / h_{1}$ increases.

Since in all the experiments conducted in this study the absolute height of the step used at the channel bottom, a, was constant, Figure 10 should not be used to explain the influence of relative step height on the values of modular limit. In order to see the effect of step height on the modular limit, similar experiments in the same channel with different step heights must be carried out and the final experimental results must be analysed. The same situation is also valid for the variation of $\left(H_{2} / H_{1}\right)_{M L}$ with $L / H_{1}$. The linear proportionality available between $a / h_{1}$ and $L / H_{1}$ results in similar relationship between $\left(H_{2} / H_{1}\right)_{M L}$ and $L / H_{1}$ as the one given in Figure 10. Due to this reason, no attempt was made to present the data of $\left(H_{2} / H_{1}\right)_{M L}$ with respect to $L / H_{1}$. 


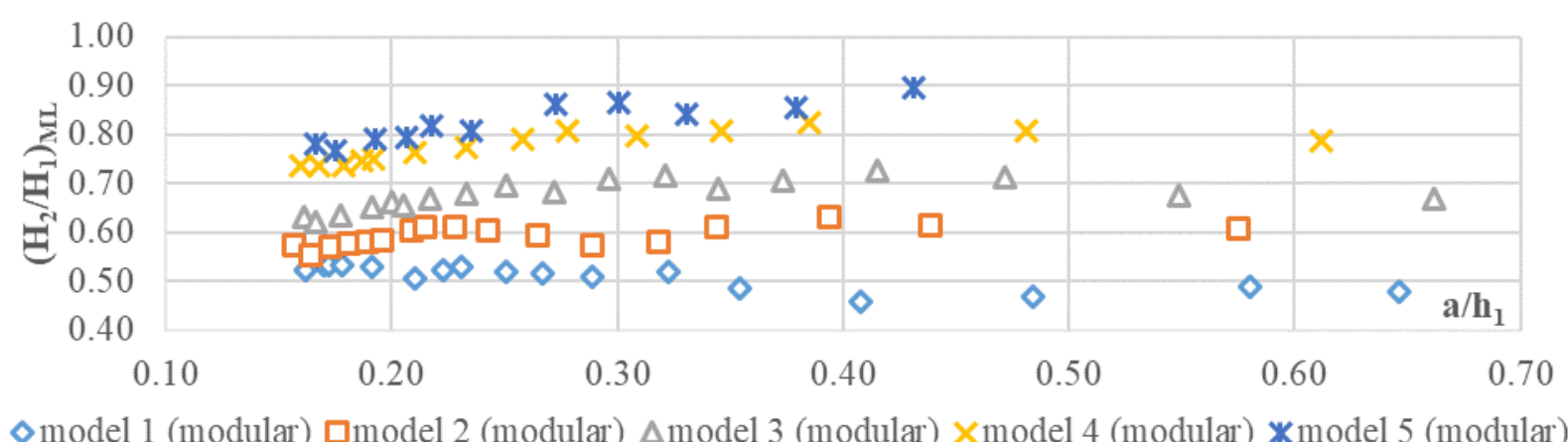

Figure 10. Variation of Modular limit with $\boldsymbol{a} / \boldsymbol{h}_{\mathbf{1}}$

\subsection{Variation of $L_{T} / h_{1}$ with Related Quantities}

In the present study, the total length of the stilling basin over which the flow becomes subcritical from supercritical was also investigated in terms of relevant parameters. From the results, one can directly determine the minimum required stilling basin length to be formed at downstream region of the structure to dissipate some part of the energy of the incoming flow.

The relationship between $L_{T} / h_{1}$ and $F r_{1}$ is presented in Figure 11 for each type of the model tested. Data points of each model show a decreasing trend with increasing $F r_{1}$ while the orders of them follow the order of the model types. $L_{T}$ value increases when $\theta$ gets smaller, so that for a given $F r_{1}$ the model types of small $\theta$ do always give high $L_{T} / h_{1}$ values.

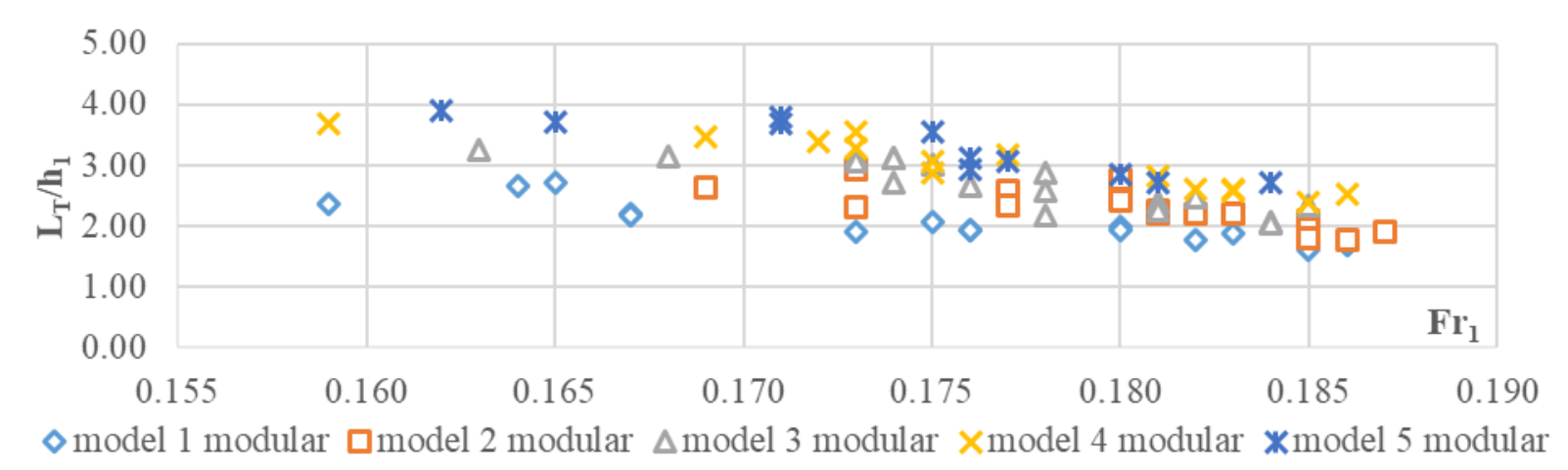

Figure 11. Variation of $\boldsymbol{L}_{T} / \boldsymbol{h}_{\mathbf{1}}$ with Froude number

In Figure 12, where the variation of $L_{T} / h_{1}$ with $a / h_{1}$ is presented, the distribution of the data are much better than those given in Figure 11. At small values of $a / h_{1}$ up to about 0.30 , it can be said that $L_{T} / h_{1}$ increases almost linearly with increasing $a / h_{1}$ for all the model types tested. The rate of change of $L_{T} / h_{1}$ with increasing $a / h_{1}$ can be considered as negligible for the values of $a / h_{1}$ larger than about 0.5.The model types of 5 and 1 give the maximum and minimum $L_{T} / h_{1}$ values, respectively, among the models tested. 


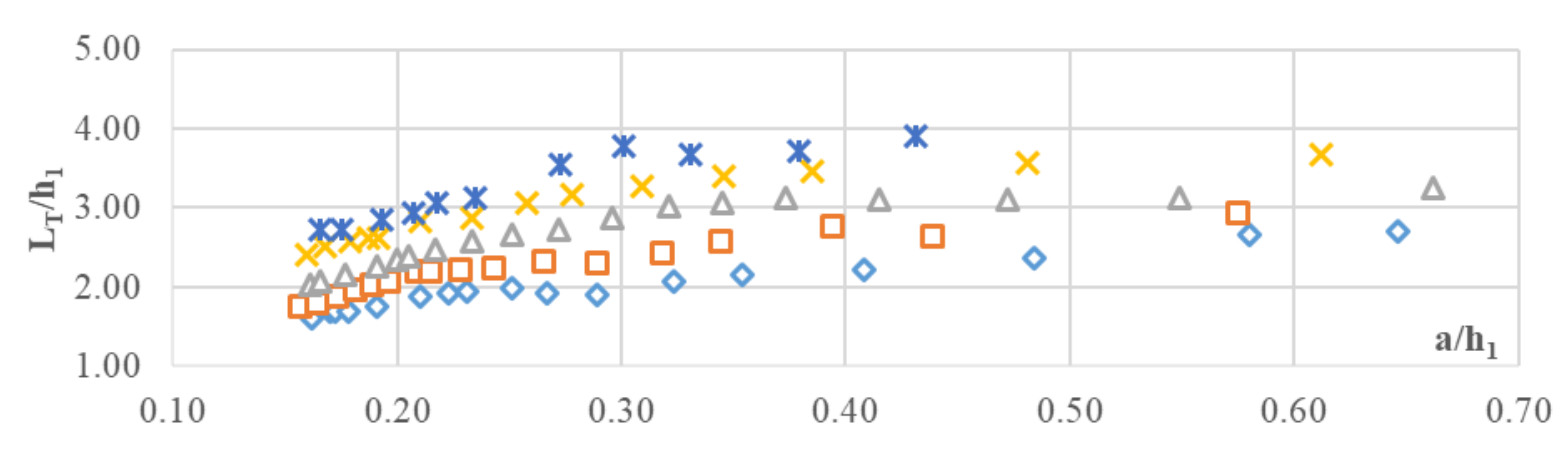

$\diamond$ model 1 modular $\square$ model 2 modular $\Delta$ model 3 modular $\times$ model 4 modular $*$ model 5 modular

Figure 12. Variation of $\boldsymbol{L}_{T} / \boldsymbol{h}_{1}$ with $\boldsymbol{a} / \boldsymbol{h}_{1}$

\section{CONCLUSIONS}

In order to investigate the effect of the type of downstream transition of a long-throated critical depth flume on the hydraulic properties of the flow, a series of laboratory experiments were conducted in this study. Following conclusions could be drawn from the analysis of the experimental results:

1) The distribution of present $C_{d}$ vs. $H_{1} / L$ data shows a similar trend as those have already been obtained in the past (Figure 3). The random distribution of the data of different model types is due to the dependency of $C_{d}$ on other dimensionless parameters such as $F r_{1}$ and $a / h_{1}$ as well as $H_{1} / L$.

2) There is a linear variation between $C_{d}$ and $F r_{1}$ data of each model type tested. The best fitting curves of these data points of the model types fall on a single line. There is no effect of the downstream transition type on the relationship between $C_{d}$ and $F r_{1}$ for the range of $F r_{1}$ investigated.

3) Within the range of $\sqrt{\alpha} C_{d} A^{*} / A_{1}$ used in the tests, the variation of this term with $C_{v}$ is almost the same for each model type tested. Therefore, it is clearly seen that the changes to be made at the downstream transition of a long-throated flume will not affect $C_{v}$ values.

4) For a given $H_{1} / L$, model type 5 yields the lowest $K_{L}$ values among the other models.

5) Modular limits, $\left(\mathrm{H}_{2} / \mathrm{H}_{1}\right)_{M L}$ slightly decrease as $\mathrm{Fr}_{1}$ increases for a given model type as seen in Figure 9 except model type 1 . This figure enables us to compute the $\left(H_{2} / H_{1}\right)_{M L}$ for a desired model and $\mathrm{Fr}_{1} ; \theta$ values smaller than that of model type $5\left(\theta \leq 41.11^{\circ}\right)$ give modular limit values of greater than 0.90 .

6) Dependency of $\left(H_{2} / H_{1}\right)_{M L}$ on $a / h_{1}$ is almost negligible, for a given model type.

7) For a given $F r_{1}, L_{T} / h_{1}$ value increases as the model type number increases (i.e. $\theta$ decreases).

\section{CONFLICTS OF INTEREST}

No conflict of interest was declared by the authors.

\section{REFERENCES}

[1] Bos, M.G., "The use of long-throated flumes to measure flows in irrigation and drainage canals", Agricultural Water Management, 1(2):111-126, (1977). https://doi.org/10.1016/03783774(77)90035-X

[2] Nashta, C.F. and Garde, R.J., "Subcritical flow in rigid-bed open channel expansions", Hydraulic Research, 26(1): 49-65, (1988). https://doi.org/10.1080/00221688809499234

[3] Swamee, P.K. and Basak, B.C., "Design of rectangular open-channel expansion transitions", Irrigation and Drainage Engineering, 117(6): 827-838, (1991). https://doi.org/10.1061/(ASCE)0733-9437(1991)117:6(827)

[4] Bhallamudi, S.M. and Chaudhry, M.H., "Computation of flows in open-channel transitions", Hydraulic Research, 30(1): 77-93, (1992). https://doi.org/10.1080/00221689209498948 
[5] Krueger, S. and Rutschmann, P., "Modelling 3D supercritical flow with extended shallow-water approach", Hydraulic Engineering, 132(9): 916-926, (2006). https://doi.org/10.1061/(ASCE)0733-9429(2006)132: 9(916)

[6] Mazumder, S.K. and Hager, W.H., "Supercritical expansion flow in rouse modified and reversed transition", Hydraulic Engineering, 119(2): 201-219, (1993). https://doi.org/10.1061/(ASCE)0733-9429(1993)119:2(201)

[7] Stamou, A.I., Chapsas, D.G. and Christodoulou, G.C., "3-D numerical modelling of supercritical flow in gradual expansions, Hydraulic Research, 46(3): 402-409, (2008). https://doi.org/10.3826/jhr.2008.3162

[8] Gogus, M., Al-Khatib, I.A. and Atalay, A.E., "Effect of the downstream transition region of a flow measurement flume of rectangular compound cross section on flow properties", Flow $\begin{array}{llll}\text { Measurement and } & \text { Instrumentation, 33(1): }\end{array}$ https://doi.org/10.1016/j.flowmeasinst.2013.05.006

[9] Nasser, A.N.N. and Li, S.S., "Reduction of flow separation and energy head losses in expansions using a hump", Irrigation and Drainage Engineering, 141(3): 04014057, (2015). https://doi.org/10.1061/(ASCE)IR.1943-4774.0000803

[10] Asnaashari, A., Akhtari, A.A., Dehghani, A.A. and Bonakdari, H., "Experimental and numerical investigation of the flow field in the gradual transition of rectangular to trapezoidal open channels", Engineering Applications of Computational Fluid Mechanics, 10(1): 272-282, (2016). https://doi.org/10.1080/19942060.2016.1149102

[11] Bos, M.G. and Reinink, Y., "Required head loss over long-throated flumes", Irrigation and Drainage Division, 107(1): 87-102, (1981). 